\title{
A QUESTION ON MCCOY RINGS
}

\author{
Zhen Lei, Jianlong Chen and Zhiling YiNg
}

\begin{abstract}
Nelsen [J. Algebra 298 (2006) 134-141] asked whether there is a natural class of McCoy rings which includes all reversible rings and all rings $R$ such that $R[X]$ is semi-commutative. In this paper, some new equivalent conditions of $\mathrm{McCoy}$ rings are given. One of them is used to answer this question in the affirmative. Finally, an example is given which is McCoy and semi-commutative, but it is not reversible and does not have the property that $R[x]$ is semi-commutative.
\end{abstract}

Throughout this paper all rings are associative with identity. For a ring $R$, let $R[x]$ be the polynomial ring and $M_{n}(R)$ be the ring of $n \times n$ matrices over $R . E_{i j}(r)$ denotes a matrix of which the $(i, j)$ entry is $r$ and others are 0 . Recall that a ring $R$ is said to be reversible if $a b=0$ implies $b a=0$ for all $a, b \in R$, and $R$ is said to be semi-commutative if $a b=0$ implies $a R b=0$ for all $a, b \in R$. In [4], a ring $R$ is called a McCoy ring if the equation $f(x) g(x)=0$, where $f(x), g(x) \in R[x] \backslash\{0\}$, implies there exist $s, t \in R \backslash\{0\}$ such that $f(x) s=\operatorname{tg}(x)=0$.

Let $\mathcal{C}$ denote the class of rings $R$ which have the property that $R$ is both McCoy and semi-commutative, and let $\mathcal{D}$ denote the class of rings $R$ which have the property that $R[x]$ is semi-commutative. In [4], Nielsen asked whether there is a natural class of $\mathrm{McCoy}$ rings which includes all reversible rings and $\mathcal{D}$. In this paper, some equivalent conditions of $\mathrm{McCoy}$ rings are proven and two examples are given to show that $\mathcal{C}$ is this natural class, giving a positive answer to the question of Nielsen. At last, an example is given which is McCoy and semi-commutative, but it is not reversible and does not have the property that $R[x]$ is semi-commutative.

More equivalent conditions of McCoy rings are proven in the following.

THEOREM 1. A ring $R$ is McCoy if and only if $R[x]$ is McCoy.

PROOF: " $\Rightarrow$ ". Let $F(y)=\sum_{i=0}^{n} f_{i} y^{i}, G(y)=\sum_{j=0}^{m} g_{j} y^{j}$ be nonzero polynomials in $R[x][y]$ such that $F(y) G(y)=0$, where $f_{i}=\sum_{s=0}^{p_{i}} a_{i s} x^{s}, g_{j}=\sum_{t=0}^{q_{j}} b_{j t} x^{t} \in R[x]$. As in the proof of

Received 28th December, 2006

This research was supported by the National Natural Science Foundation of China (No.10571026), the Natural Science Foundation of Jiangsu Province (No.2005207), the Cultivation Fund of the Key Scientific and Technical Innovation Project of Ministry of Education (China), and the Specialized Research Fund for the Doctoral Program of Higher Education (20060286006).

Copyright Clearance Centre, Inc. Serial-fee code: 0004-9727/07 \$A2.00+0.00. 
[1, Theorem 2], we let $k=\sum \operatorname{deg} f_{i}+\sum \operatorname{deg} g_{j}$, where the degree is as polynomial in $x$ and the degree of the zero polynomial is taken to be 0 . Then $F\left(x^{k}\right)=\sum_{i=0}^{n} f_{i} x^{i k}$, $G\left(x^{k}\right)=\sum_{j=0}^{m} g_{j} x^{j k} \in R[x]$ and the set of coefficients of the $f_{i}^{\prime} s$ (respectively $g_{j}^{\prime} s$ ) equals the set of coefficients of $F\left(x^{k}\right)$ (respectively $G\left(x^{k}\right)$ ). Since $F(y) G(y)=0$ and $x$ commutes with elements of $R, F\left(x^{k}\right) G\left(x^{k}\right)=0$. Because $R$ is McCoy, there exist $u, v \in R \backslash\{0\}$ such that $F\left(x^{k}\right) u=0, v G\left(x^{k}\right)=0$. Thus, $F(y) u=0, v G(y)=0$. That is, $R[x]$ is McCoy.

" $\Leftarrow$ ". Let $f(x)=\sum_{i=0}^{n} a_{i} x^{i}, g(x)=\sum_{j=0}^{m} b_{j} x^{j} \in R[x] \backslash\{0\}$ such that $f(x) g(x)=0$. Let $f(y)=\sum_{i=0}^{n} a_{i} y^{i}, g(y)=\sum_{j=0}^{m} b_{j} y^{j}$. Then $f(y), g(y) \in R[x][y]$ and $f(y) g(y)=0$. Since $R[x]$ is McCoy, there exists $h(x)=\sum_{k=0}^{p} h_{k} x^{k} \in R[x] \backslash\{0\}$ such that $f(y) h(x)=0$. Hence $a_{i} h(x)=0$ for every $i=0,1, \ldots n$. Thus, there exists $h_{k} \in R \backslash\{0\}$ such that $f(x) h_{k}=0$. Similarly, there exits $t \in R \backslash\{0\}$ such that $t g(x)=0$. Hence $R$ is McCoy.

ThEOREM 2. A ring $R$ is McCoy if and only if the ring

$$
R_{n}=\left\{\left(\begin{array}{ccccc}
a & a_{12} & a_{13} & \ldots & a_{1 n} \\
0 & a & a_{23} & \ldots & a_{2 n} \\
0 & 0 & a & \ldots & a_{3 n} \\
\vdots & \vdots & \vdots & \ddots & \vdots \\
0 & 0 & 0 & \ldots & a
\end{array}\right): a, a_{k l} \in R\right\}
$$

is McCoy for any $n \geqslant 1$.

Proof: “ $\Rightarrow$ ". Let

$$
A_{i}=\left(\begin{array}{cccc}
a_{i} & a_{12}^{(i)} & \ldots & a_{1 n}^{(i)} \\
0 & a_{i} & \ldots & a_{2 n}^{(i)} \\
\vdots & \vdots & \ddots & \vdots \\
0 & 0 & \ldots & a_{i}
\end{array}\right), B_{j}=\left(\begin{array}{cccc}
b_{j} & b_{12}^{(j)} & \ldots & b_{1 n}^{(j)} \\
0 & b_{j} & \ldots & b_{2 n}^{(j)} \\
\vdots & \vdots & \ddots & \vdots \\
0 & 0 & \ldots & b_{j}
\end{array}\right) \in R_{n}
$$

Then

$$
\begin{aligned}
& F(x)=\sum_{i=0}^{p} A_{i} x^{i}=\left(\begin{array}{cccc}
f(x) & f_{12}(x) & \ldots & f_{1 n}(x) \\
0 & f(x) & \ldots & f_{2 n}(x) \\
\vdots & \vdots & \ddots & \vdots \\
0 & 0 & \ldots & f(x)
\end{array}\right), \\
& G(x)=\sum_{j=0}^{q} B_{j} x^{j}=\left(\begin{array}{cccc}
g(x) & g_{12}(x) & \ldots & g_{1 n}(x) \\
0 & g(x) & \ldots & g_{2 n}(x) \\
\vdots & \vdots & \ddots & \vdots \\
0 & 0 & \ldots & g(x)
\end{array}\right),
\end{aligned}
$$


where $f(x)=\sum_{i=0}^{p} a_{i} x^{i}, f_{k l}(x)=\sum_{i=0}^{p} a_{k l}^{(i)} x^{i}, g(x)=\sum_{j=0}^{q} b_{j} x^{j}, g_{k l}(x)=\sum_{j=0}^{q} b_{k l}^{(j)} x^{j}$ for any $k=1,2, \ldots, n, l=2,3, \ldots, n$ and $k<l$.

Suppose $F(x) G(x)=0$, and $F(x), G(x) \neq 0$. Set $H(x)=F(x) G(x)=\left(h_{p q}(x)\right)$ for $p, q=1,2, \ldots, n$.

CASE 1. If $f(x) \neq 0, g(x) \neq 0$, then $h_{11}(x)=f(x) g(x)=0$. Since $R$ is McCoy, there exist $s, t \in R \backslash\{0\}$ such that $f(x) s=0$, and $t g(x)=0$. Let $A=E_{1 n}(s), B=E_{1 n}(t)$. Then $F(x) A=0$ and $B G(x)=0$.

CASE 2. If $f(x) \neq 0, g(x)=0$, then there exists $g_{k l}(x) \neq 0$, such that $g_{k+u, l}(x)=0$ for some $k, l$, and $1 \leqslant u \leqslant n-k$ since $G(x) \neq 0$. So $h_{k l}(x)=f(x) g_{k l}(x)=0$. There exists $s \in R \backslash\{0\}$ such that $f(x) s=0$ because $R$ is McCoy. Let $A=E_{1 n}(s)$. Then $F(x) A=A G(x)=0$.

CASE 3. If $f(x)=0, g(x) \neq 0$, then there exist $A, B \in R_{n} \backslash\{0\}$ such that $F(x) A$ $=B G(x)=0$. The proof is similar to Case 2 .

CASE 4. If $f(x)=0, g(x)=0$, then for any $s \in R \backslash\{0\}$, let $A=E_{1 n}(s)$. It is obvious that $F(x) A=A G(x)=0$.

Therefore, $R_{n}$ is McCoy for any case.

" $\Leftarrow$ ". Assume that $f(x) g(x)=0$, where

$$
f(x)=\sum_{i=0}^{n} a_{i} x^{i} \neq 0, g(x)=\sum_{j=0}^{m} b_{j} x^{j} \neq 0, a_{i}, b_{j} \in R .
$$

Let $F(x)=\sum_{i=0}^{n} A_{i} x^{i}, G(x)=\sum_{j=0}^{m} B_{j} x^{j}$, where

$$
A_{i}=\left(\begin{array}{cccc}
a_{i} & a_{i} & \ldots & a_{i} \\
0 & a_{i} & \ldots & a_{i} \\
\vdots & \vdots & \ddots & \vdots \\
0 & 0 & \ldots & a_{i}
\end{array}\right), B_{j}=\left(\begin{array}{cccc}
b_{j} & b_{j} & \ldots & b_{j} \\
0 & b_{j} & \ldots & b_{j} \\
\vdots & \vdots & \ddots & \vdots \\
0 & 0 & \ldots & b_{j}
\end{array}\right)
$$

for any $i=0,1, \ldots n, j=0,1, \ldots m$. Then

$$
F(x) G(x)=\left(\begin{array}{cccc}
f(x) & f(x) & \ldots & f(x) \\
0 & f(x) & \ldots & f(x) \\
\vdots & \vdots & \ddots & \vdots \\
0 & 0 & \ldots & f(x)
\end{array}\right)\left(\begin{array}{cccc}
g(x) & g(x) & \ldots & g(x) \\
0 & g(x) & \ldots & g(x) \\
\vdots & \vdots & \ddots & \vdots \\
0 & 0 & \ldots & g(x)
\end{array}\right)=0
$$

Hence, there exists $A=\left(\begin{array}{cccc}s & s_{12} & \ldots & s_{1 n} \\ 0 & s & \ldots & s_{2 n} \\ \vdots & \vdots & \ddots & \vdots \\ 0 & 0 & \ldots & s\end{array}\right) \in R_{n} \backslash\{0\}$ such that $F(x) A=0$ because $R_{n}$ is McCoy. If $s \neq 0$, then $f(x) s=0$. If $s=0$, then there exists $s_{i j} \neq 0$ for some $i, j$, such 
that $s_{i+v, j}=0$ for any $1 \leqslant v \leqslant n-i$. We also have $f(x) s_{i j}=0$. Similarly, there exists $t \in R \backslash\{0\}$ such that $t g(x)=0$. Thus, $R$ is McCoy.

Example 3. Let $R$ be a McCoy ring. By Theorem $2, R_{4}$ is McCoy. Since $E_{12}(1) E_{34}(1)$ $=0$ and $E_{12}(1) E_{23}(1) E_{34}(1) \neq 0, R_{4}$ is not semi-commutative.

REMARK 4. In [2, Theorem 2], it is proven that $R$ is McCoy if $R \in \mathcal{D}$. It is obvious that the subring of a semi-commutative ring is also. So that $R[x]$ is semi-commutative implies $R$ is semi-commutative. Thus, $\mathcal{D} \subseteq \mathcal{C}$. Nielsen proven that all reversible rings are $\mathrm{McCoy}$ in [4], and it is a known result that a reversible ring is semi-commutative. So $\mathcal{C}$ includes the class of all reversible rings. Moreover, there is an example of a ring that is semi-commutative but not McCoy in [4], and $R_{4}$ in Example 3 is McCoy but not semi-commutative. Hence, $\mathcal{C}$ is a natural class of McCoy rings (and of semi-commutative rings) which includes all reversible rings and $\mathcal{D}$. It gives a positive answer to the question of Nielsen in [4].

The following example is constructed by Huh, Lee and Smoktunowicz in [3, Example 2]. We further investigate more properties of the ring in this example.

EXAMPLE 5. Let $Z_{2}$ be the field of integers modulo 2 and $A=Z_{2}\left[a_{0}, a_{1}, a_{2}, b_{0}, b_{1}, b_{2}, c\right]$ be the free algebra of polynomials with zero constant terms in noncommuting indeterminates $a_{0}, a_{1}, a_{2}, b_{0}, b_{1}, b_{2}, c$ over $Z_{2}$. Note that $A$ is a ring without identity and consider an ideal $I$ of $Z_{2}+A$ generated by $a_{0} b_{0}, a_{1} b_{2}+a_{2} b_{1}, a_{0} b_{1}+a_{1} b_{0}, a_{0} b_{2}+a_{1} b_{1}$ $+a_{2} b_{0}, a_{2} b_{2}, a_{0} r b_{0}, a_{2} r b_{2},\left(a_{0}+a_{1}+a_{2}\right) r\left(b_{0}+b_{1}+b_{2}\right)$ with $r \in A$ and $r_{1} r_{2} r_{3} r_{4}$ with $r_{1}, r_{2}, r_{3}, r_{4} \in A$. Let $R=\left(Z_{2}+A\right) / I$. Then $R$ is a semi-commutative and McCoy ring. But $R$ is not reversible, and $R[x]$ is not semicommutative.

Proof: It was proven that $R$ is semicommutative but $R[x]$ does not have the same property in [3]. $R$ is not reversible since $a_{0} b_{0} \in I$ but $b_{0} a_{0} \notin I$. We use the notion as [3, Example 2]. Each product of indeterminates $a_{0}, a_{1}, a_{2}, b_{0}, b_{1}, b_{2}, c$ is called a monomial and $\alpha$ is a monomial of degree $n$ if it is a product of exactly $n$ generators. Let $H_{n}$ be the set of all linear combinations of monomials of degree $n$ over $Z_{2}$. Then $H_{n}$ is finite for any $n$ and $I$ is homogeneous (that is, if $\sum_{i=1}^{n} r_{i} \in I$ with $r_{i} \in H_{i}$ then every $r_{i} \in I$ ).

Write $\bar{r}=r+I \in R$ for every $r \in Z_{2}+A$. Since $A^{4} \subseteq I, \bar{r}$ can be written in the form $\bar{r}=\bar{a}+\bar{h}_{1}+\bar{h}_{2}+\bar{h}_{3}$ with $a \in Z_{2}, h_{i} \in H_{i}(i=1,2,3)$. Write $f(x)=\sum_{i=0}^{n} \bar{p}_{i} x^{i}$ and $g(x)=\sum_{j=0}^{m} \bar{q}_{j} x^{j}$, where $\bar{p}_{i}=\bar{a}_{p}^{(i)}+\bar{f}_{1}^{(i)}+\bar{f}_{2}^{(i)}+\bar{f}_{3}^{(i)}, \bar{q}_{j}=\bar{a}_{q}^{(j)}+\bar{g}_{1}^{(j)}+\bar{g}_{2}^{(j)}+\bar{g}_{3}^{(j)}$ for some $a_{p}^{(i)}, a_{q}^{(j)} \in Z_{2}$ and $f_{k}^{(i)}, g_{k}^{(j)} \in H_{k}, i=0,1, \ldots, n, j=0,1, \ldots, m, k=1,2,3$.

ClAIM. Suppose that $f(x) g(x)=0$. If not all $\bar{a}_{p}^{(i)}=0$, then $g(x)=0$. If not all $\bar{a}_{q}^{(j)}=0$, then $f(x)=0$.

Suppose that $s$ is the smallest index such that $\bar{a}_{p}^{(s)} \neq 0$. That is to say $\bar{a}_{p}^{(s)}=\overline{1}$. If not all $\bar{a}_{q}^{(j)}=0$, then we can assume that $j_{1}$ is the smallest index such that $\bar{a}_{q}^{\left(j_{1}\right)}=\overline{1}$. Compare 
the coefficient of $x^{\left(s+j_{1}\right)}$ in $f(x) g(x)=0$. Then there exist $h_{i} \in H_{i}(i=1,2,3)$ such that $\bar{a}_{p}^{(s)} \bar{a}_{q}^{\left(j_{1}\right)}+\bar{h}_{1}+\bar{h}_{2}+\bar{h}_{3}=0$. Thus, $\bar{h}_{1}+\bar{h}_{2}+\bar{h}_{3}=\overline{1}$, a contradiction. Hence all $\bar{a}_{q}^{(j)}=0$. The Claim is proven by induction on $k$. Suppose that all $\bar{g}_{k-1}^{(j)}=0$ for $k \leqslant 3$. Next we prove $\bar{g}_{k}^{(j)}=0$ for every $j$. Assume the contrary. Then we can find the smallest index $j_{2}$ such that $\bar{g}_{k}^{\left(j_{2}\right)} \neq 0$. From the equation $f(x) g(x)=0$, there exist $h_{k+1}^{\prime} \in H_{k+1}, \ldots, h_{3}^{\prime} \in H_{3}$ such that $\bar{g}_{k}^{\left(j_{2}\right)}+{\overline{h^{\prime}}}_{k+1}+\cdots+{\overline{h^{\prime}}}_{3}=0$. that is, $g_{k}^{\left(j_{2}\right)}+h_{k+1}^{\prime}+\cdots+h_{3}^{\prime} \in I$. Because $I$ is homogeneous, $g_{k}^{\left(j_{2}\right)} \in I$. This leads to a contradiction. Hence $g(x)=0$. The remain is similar to proof.

Let $f(x), g(x) \in R[x] \backslash\{0\}$ with $f(x) g(x)=0$. By the Claim, $\bar{a}_{p}^{(i)}=\bar{a}_{q}^{(j)}=0$ for every $i, j$. Choose $t=\bar{c}^{3}$. Then $t \neq 0$ and $f(x) t=t g(x)=0$. Therefore, $R$ is a McCoy ring.

REMARK $6 . \quad$ Example 5 shows that $\mathcal{C}$ properly includes the class of reversible rings and $\mathcal{D}$. The following implications hold.

$$
\begin{aligned}
& R \in \mathcal{D} \\
& \Downarrow \\
& R \text { is reversible } \quad \Rightarrow \quad R \in \mathcal{C} \quad \Rightarrow \quad R \text { is semi-commutative } \\
& \Downarrow \\
& R \text { is } \mathrm{McCoy}
\end{aligned}
$$

In the table above, each of these implications is irreversible, and there are no other implications between these conditions.

\section{REFERENCES}

[1] D.D. Anderson and V. Camillo, 'Armendariz rings and Gaussian rings', Comm. Algebra 26 (1998), 2265-2272.

[2] Y. Hirano, 'On annihilator ideals of a polynomial ring over a noncommutative ring', $J$. Pure Appl. Algebra 168 (2002), 45-52.

[3] C. Huh, Y. Lee and A. Smoktunowicz, 'Armendariz rings and semicommutative rings', Comm. Algebra 30 (2002), 751-761.

[4] P.P. Nielsen, 'Semi-commutativity and the McCoy condition', J. Algebra 298 (2006), 134-141.

Department of Mathematics

Anhui Normal University

Wuhu 241000

Peoples Republic of China

e-mail: wuhuleizhen@126.com

\author{
Department of Mathematics \\ Southeast University \\ Nanjing 210096 \\ Peoples Republic of China \\ e-mail: jlchen@seu.edu.cn \\ e-mail: zhilingying@yahoo.com.cn
}

